\title{
Preparation of Graphene Quantum Dots and Their Sensing Properties in Quartz Crystal Microbalance Acetone Sensor
}

\author{
Jing Wang, Suhua Lin, Yilin Lin, and Xiaohua Wang* \\ East China Normal University, 500 Dongchuan Road, Minhang District, Shanghai 201100, China
}

(Received August 27, 2020; accepted December 15, 2020)

Keywords: graphene quantum dots, citrate pyrolysis, acetone, QCM

Acetone is a slightly toxic volatile organic gas, which exists in the breath and is closely related to diseases such as diabetes. In this paper, a quartz crystal microbalance (QCM) was used to fabricate an acetone sensor, and graphene quantum dots (GQDs) were used as a gas-sensing material to modify the QCM. GQDs were prepared by citrate pyrolysis and characterized by high-resolution transmission electron microscopy (TEM). The gas sensitivity of the sensor to low concentrations of acetone was investigated. It exhibited good linearity at acetone concentrations of less than $240 \mathrm{ppm}$ with a sensitivity of $16.78 \mathrm{~Hz} / \mathrm{ppm}$ and a minimum detection limit of $2.5 \mathrm{ppm}$, and the fitted line had a coefficient of determination $R^{2}$ of 0.95658 . In a mixture of acetone, butanol, and isopropanol, the sensor exhibited good selectivity for acetone. For different acetone concentrations, the response speed of the same sensor was basically the same, and the response and recovery times were 32 and $48 \mathrm{~s}$, respectively. We showed that the prepared gas sensor has good sensitivity, repeatability, and selectivity for low concentrations of acetone.

\section{Introduction}

With the acceleration of the pace of life, the air pollution caused by human activities is gradually increasing. The indoor pollutants are mainly volatile organic compounds (VOCs) such as formaldehyde, acetone, and benzene. Among them, although acetone is not carcinogenic like formaldehyde, it is slightly toxic. ${ }^{(1)}$ Its main harmful effects are anesthesia and inhibition of the central nervous system. Long-term exposure to an environment with acetone content higher than 1\% may cause headaches, vomiting, and even coma. In addition, according to recent research findings, the detection of VOCs in exhaled breath can be used to determine certain diseases. There is a close relationship between acetone and diabetes. Studies have shown that the level of acetone in exhaled breath can be used to determine whether a user has diabetes. ${ }^{(2)}$

The above background has driven the development of simple and efficient gas detection tools such as gas sensors. At present, common methods for measuring acetone gas include chromatography, a light-sensing method, and a spectrophotometric method. These methods

*Corresponding author: e-mail: xhwang@ee.ecnu.edu.cn https://doi.org/10.18494/SAM.2021.3075 
generally have problems of complicated operation, expensive equipment, and difficulty of realtime measurement.

Among the acetone gas sensors that have been studied so far, conventional semiconductor gas sensors based on metal oxides such as $\mathrm{SnO}_{2}, \mathrm{WO}_{3}, \mathrm{ZnO}$, and $\mathrm{In}_{2} \mathrm{O}_{3}$ have insufficient sensitivity and selectivity, ${ }^{(3-8)}$ and need to operate at high temperatures. In 2017, Xu et al. ${ }^{(9)}$ studied a highly sensitive VOC-acetone sensor based on Ag-modified $\mathrm{SnO}_{2}$ hollow nanofibers, which has high responsiveness and selectivity to acetone at $160{ }^{\circ} \mathrm{C}$. In the same year, Jiang et al. ${ }^{(10)}$ studied the application of $\mathrm{Ca}^{2+} / \mathrm{Au}$-doped $\mathrm{SnO}_{2}$ nanofibers in acetone sensors with high responsivity at $180{ }^{\circ} \mathrm{C}$. In 2018 , Preethi et al. ${ }^{(11)}$ found that $1 \%$ Ga-doped thin films were an effective acetone gas sensor with $68 \%$ sensitivity at $350{ }^{\circ} \mathrm{C}$. In 2019 , Ming-Jiang and Bing-Ying ${ }^{(12)}$ prepared copper oxide/zinc oxide $(\mathrm{CuO} / \mathrm{ZnO})$ nanostructured flowers by a lowtemperature hydrothermal method. Their newly designed thin-film acetone gas sensor showed a wide linear range $\left(10-300 \mathrm{mg} / \mathrm{cm}^{3}\right.$ acetone) at a working temperature of $200{ }^{\circ} \mathrm{C}$. In the same year, He et al. ${ }^{(13)}$ synthesized $\mathrm{Pd}-\mathrm{WO}_{3}$ nanosheets through a one-step hydrothermal method using $\mathrm{Na}_{2} \mathrm{PdCl}_{4}$ solution as the palladium source and sodium tungstate as the tungsten source, and used them to detect acetone. The detection limit for acetone was $50 \mathrm{ppb}$ at the optimum working temperature of $300{ }^{\circ} \mathrm{C}$, and the selectivity for acetone was excellent in a $100 \mathrm{ppm}$ acetone atmosphere.

There have also been some studies on acetone sensors operating at room temperature (RT). A quartz crystal microbalance (QCM) sensor is a mass detection platform based on the piezoelectric effect of a quartz crystal, which is very sensitive to changes in quality, humidity, density, and conductivity. A QCM has nanogram sensitivity, allowing it to detect microscopic changes and acquire large amounts of data in real time. A gas sensor based on the QCM sensing structure has the advantages of high availability, strong robustness, high versatility, small size, strong anti-interference, and high stability. The Vietnamese research team of Quang et al. ${ }^{(14)}$ used chemical vapor deposition to grow graphene on a QCM in 2014. At RT, the response value $\Delta f$ of the prepared sensor to $100 \mathrm{ppm}$ acetone gas was $14.8 \mathrm{~Hz}$, and its response and recovery time were 42 and $59 \mathrm{~s}$, respectively. In 2016, Tao et al. ${ }^{(15)}$ prepared a composite material of $\mathrm{TiO}_{2}$ and multiwalled carbon nanotubes (MWCNTs, an allotrope of carbon) as a sol-gel and spincoated it on a QCM wafer. The minimum detection limit of this sensor was 4.33 ppmv.

The main component of graphene quantum dots (GQDs) is carbon, which is a quantum dot material with low toxicity and environmental impact. As a quasi-zero-dimensional nanomaterial, GQDs not only possess the unique properties of graphene, but also have many excellent physical and chemical properties due to their remarkable quantum confinement effect and boundary effect. ${ }^{(16)}$ GQDs have good biocompatibility, band gap adjustability, a large specific surface area, low toxicity, and good water solubility. ${ }^{(17)}$ In general, there are two major routes for synthesizing GQDs: top-down and bottom-up. Top-down methods include electron beam lithography, acid oxidative stripping, microwave assistance, hydrothermal synthesis, and electrochemical oxidation. ${ }^{(18-21)}$ This preparation route has the advantages of abundant raw materials, simple operation, and mass production and the disadvantages of high requirements in terms of equipment, easy destruction of the aromatic structure of graphite, and the incapability of accurately controlling the form and size of the synthesized product. The bottom-up 
mechanism involves the assembly of small molecules into relatively large GQDs, with methods including the pyrolysis of polycyclic aromatic hydrocarbons, the use of a carbonized organic precursor, and solution chemistry. ${ }^{(22-24)}$ The advantage of this preparation route is better control of the form, size, and related properties of the synthesized product. Its disadvantages are that the operation is complicated, the raw materials of the precursor are difficult to obtain, and the solubility of the product is poor.

In this study, GQDs were prepared by a citrate pyrolysis method, and the morphology of the material samples was characterized by transmission electron microscopy (TEM). We used GQDs as the sensitive material for gas sensors and tested the gas-sensing properties of the sensor for acetone gas at RT.

\section{Experiment}

\subsection{Chemical reagents and equipment}

The chemical reagents used in this experiment were of analytical grade. Citric acid (CA) was purchased from Tianjin Dingshengxin Chemical Co., Ltd., sodium hydroxide and nitric acid were purchased from Sinopharm Holding (Shanghai) Chemical Reagent Co., Ltd., and deionized water was made in our laboratory. No reagents were subjected to further processing prior to use. The instruments and materials we used included a CHI400C series QCM, digital thermostat magnetic stirrer, ultraviolet analyzer, high-precision electronic analytical balance, vacuum drying oven, ultrasonic cleaning instrument, $\mathrm{pH}$ test paper, pipetting gun, temperature and humidity meter, and MD55-3500 dialysis bag.

\subsection{Preparation of GQDs by citrate pyrolysis}

Using CA as the carbon source, dehydration condensation of CA molecules was achieved by pyrolysis carbonization to form GQDs. The procedure was as follows. Firstly, $2 \mathrm{~g}$ of CA was placed in a $50 \mathrm{ml}$ round-bottom flask, which was then placed on a $200{ }^{\circ} \mathrm{C}$ constant-temperature heating platform for heating. After $3 \mathrm{~min}$, the CA crystals gradually began to liquefy. We observed that the liquid changed from colorless to pale yellow to orange after heating for $10 \mathrm{~min}$. Secondly, the thus obtained orange liquid was added to $100 \mathrm{ml}$ of $10 \mathrm{mg} / \mathrm{ml} \mathrm{NaOH}$ solution, and the mixture was magnetically stirred vigorously for about $30 \mathrm{~min}$. Thirdly, 1:10 dilute nitric acid was used to adjust the solution to a neutral $\mathrm{pH}$. Finally, the obtained GQD solution was stored in a refrigerator at $4{ }^{\circ} \mathrm{C}$ for future use. The principle of pyrolyzing CA to prepare GQDs is shown in Fig. 1. Furthermore, for subsequent characterization, a small amount of GQDs was subjected to dialysis purification treatment for 1-2 days using an MD55-3500 dialysis bag.

\subsection{Sample characterization}

A small amount of the GQD solution prepared by the above method was poured into a clean centrifuge tube. In order to see the result more clearly, we placed three centrifuge tubes together 


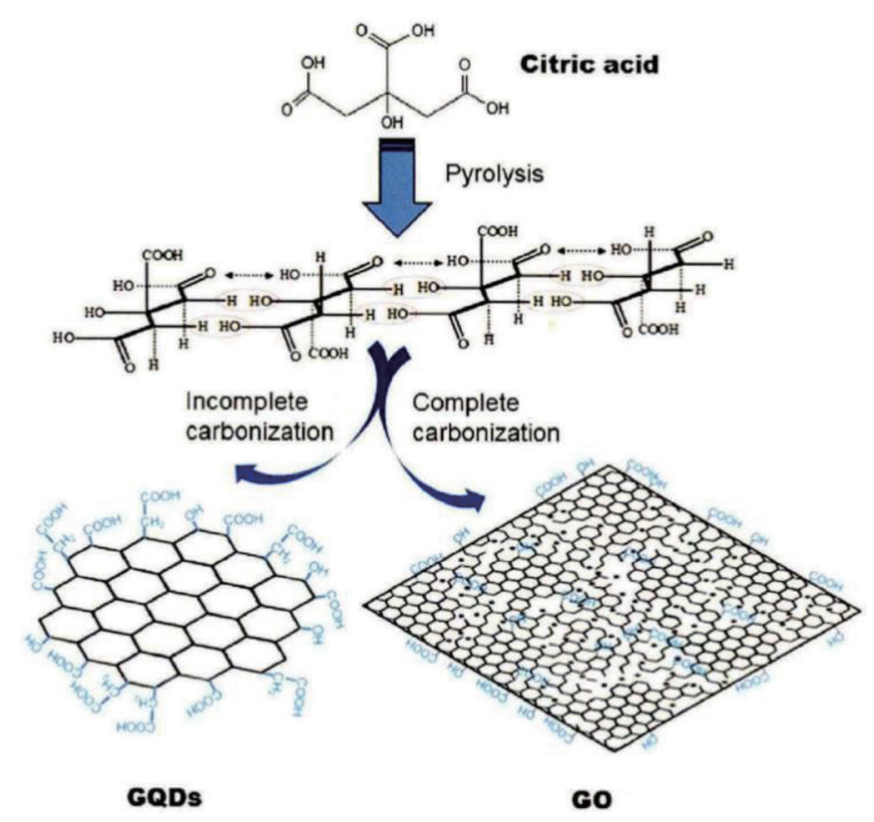

Fig. 1. (Color online) Schematic diagram of preparing GQDs by pyrolysis of CA.

under an ultraviolet analyzer for comparison. An empty centrifuge tube without any liquid was placed on the left, a centrifuge tube with deionized water was placed in the middle, and a centrifuge tube with the GQD solution was placed on the right. It can be clearly seen from Fig. 2 that the rightmost tube emitted blue fluorescence under $365 \mathrm{~nm}$ ultraviolet radiation. From this phenomenon, we could preliminarily judge that we had successfully prepared GQDs emitting blue fluorescence.

TEM was used to observe the morphology of microstructures smaller than $0.2 \mu \mathrm{m}$. The test equipment we used here was a JEM-2100F high-resolution transmission electron microscope provided by the School Electron Microscope Center. Figure 3 shows TEM images of GQDs prepared by heating $\mathrm{CA}$ at $200{ }^{\circ} \mathrm{C}$ for $10-15 \mathrm{~min}$. As can be seen from Figs. 3(a)-3(c), the GQDs are basically encapsulated in organic matter, their morphology is roughly spherical or elliptical, and most of the GQDs have a particle size of about $10 \mathrm{~nm}$. Moreover, we can see from the highresolution TEM image [Fig. 3(d)] that the material shows a clean lattice fringe, which means that it has good crystallinity.

\subsection{Production of QCM gas sensor}

A CHI400C series electrochemical QCM was purchased from Shanghai Chenhua Instrument Co., Ltd. It included a frequency counter, quartz crystal oscillator, signal filter, fast digital signal generator, and other modules. Its maximum data acquisition rate was $1 \mathrm{MHz}$. The quartz wafers used in this experiment were gold-plated electrodes with a fundamental frequency of $8 \mathrm{MHz}$ and a disk diameter of $14 \mathrm{~mm}$. 


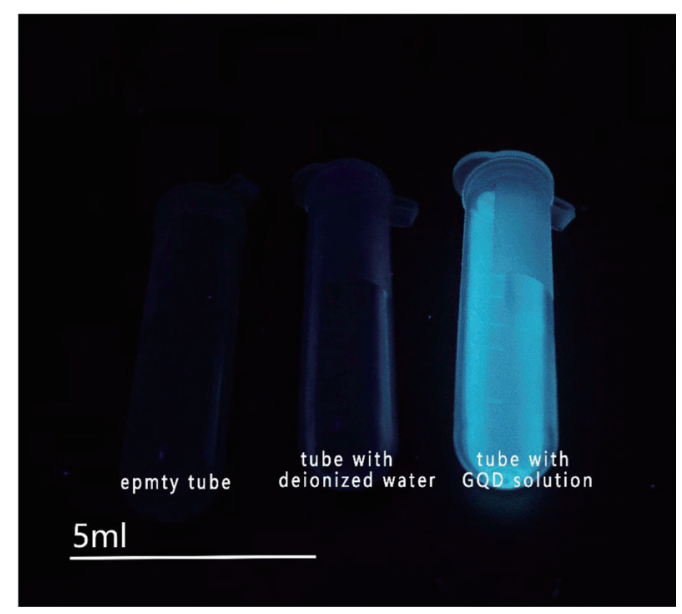

Fig. 2. (Color online) Blue fluorescence under $365 \mathrm{~nm} \mathrm{UV}$ irradiation.

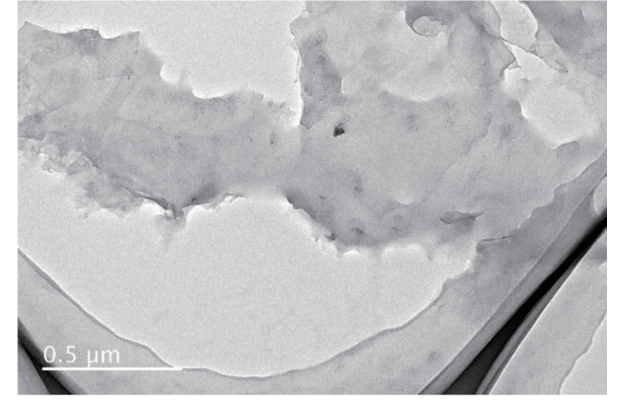

(a)

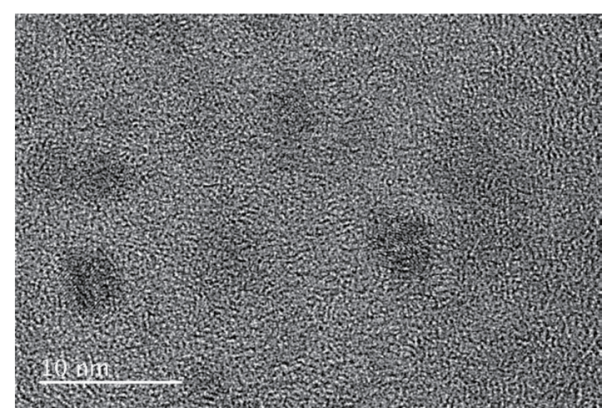

(c)

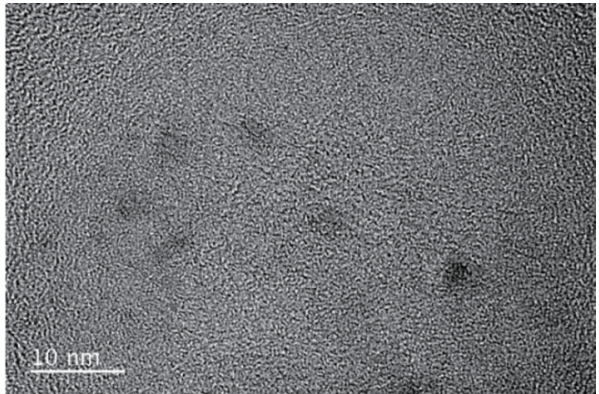

(b)

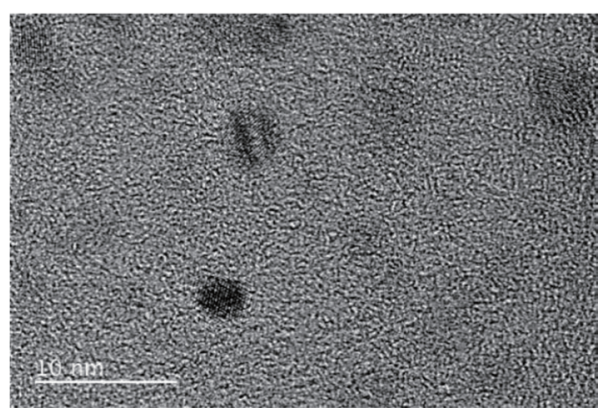

(d)

Fig. 3. TEM images of GQDs. (a) TEM image of GQDs under $0.5 \mu \mathrm{m}$ scale. (b) TEM image of GQDs under $10 \mathrm{~nm}$ scale. (c) TEM image of GQDs under $10 \mathrm{~nm}$ scale. (d) HR-TEM image of GQDs.

\subsubsection{Wafer cleaning}

Since a wafer usually has contaminants before use such as particles, metals, and organic substances, each wafer was first chemically cleaned, and then its surface was hydrophilized. Here, concentrated sulfuric acid/hydrogen peroxide was used as the cleaning solution. After natural drying, we measured the frequency of the bare wafer and used this as the reference frequency of the wafer. 


\subsubsection{Material coating and film thickness calculation}

We used a pipette to draw the GQD solution onto the quartz wafer and tried to spread the droplets evenly over the surface of the wafer. The wafer was kept level and stored in a dry box for $12 \mathrm{~h}$. After drying, there was a uniform film on the surface of the wafer. Finally, we measured and recorded the wafer frequency.

After obtaining the vibration frequency of the sensor before and after coating the material, the electrode area and film thickness were calculated using the Sauerbrey formula:

$$
\Delta f=-2.26 \times 10^{-6} f_{0}^{2} \Delta M / A,
$$

which can be converted to

$$
\Delta M=-\frac{A \Delta f}{2.26 \times 10^{-6} f_{0}^{2}} .
$$

Here, we assumed that the sensitive membrane material was ideally uniform and rigidly applied to the surface of the wafer, and from the above formula, we calculated the quality of the coating $(\Delta M)$. From the coating density $\rho_{t}\left(\mathrm{~g} / \mathrm{cm}^{3}\right)$, the thickness of the coating can be derived as

$$
\begin{gathered}
T_{t}=\Delta M / A \rho_{t}, \\
T_{t}=-\frac{\Delta f}{2.26 \times 10^{-6} f_{0}^{2} \rho_{t}} .
\end{gathered}
$$

In the calculation, we assumed a density of $2.257 \mathrm{~g} / \mathrm{cm}^{3}$, and the calculated result was only used as a rough estimate for comparison. Through the above calculations, we obtained the film thickness through the frequency difference before and after coating the material for subsequent experiments.

\subsection{Gas-sensing detection device and system calibration}

The components of a gas detection device generally include a gas distribution platform, QCM, sensor signal detection circuit, and QCM output data processing software. Gas flowmeters and gas valves were used in the experiment to control the generation and delivery of gas. A schematic diagram of the test system is shown in Fig. 4. The following tests were carried out at RT $\left(25^{\circ} \mathrm{C}\right)$ with the relative humidity controlled at $30 \%$. The main characteristic parameters measured to evaluate the performance of QCM gas sensors are sensitivity, stability, repeatability, response and recovery times, and selectivity. The following experiments were carried out to determine these performance indicators.

Five wafers were selected for this gas-sensing experiment, and each wafer was coated with a sensitive film of a different thickness. The film thickness was calculated according to the above Sauerbrey formula. For the follow-up research, we numbered the wafers coated with different film thicknesses from \#1 to \#5 in order of increasing thickness, as shown in Table 1. 


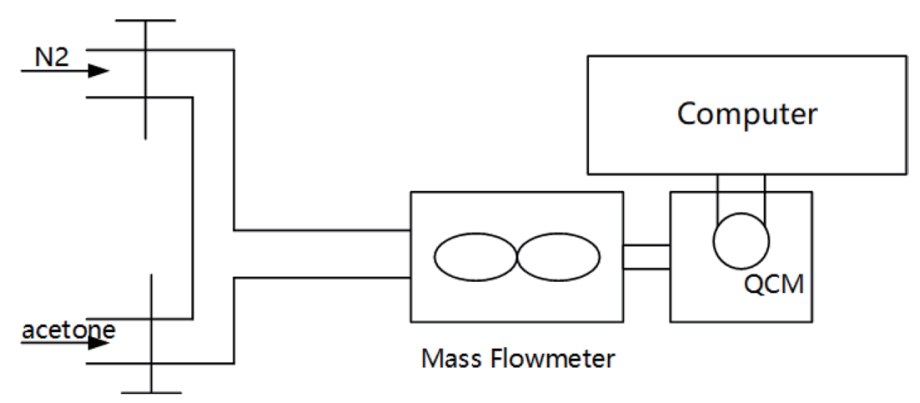

Fig. 4. Schematic of gas detection device.

Table 1

Sample numbers, film thicknesses, and parameter values.

\begin{tabular}{lcccc}
\hline Number & Reference frequency & Post-coating frequency & Change & Thickness \\
\hline$\# 1$ & 7995809 & 7990316 & 5493 & 168 \\
\#2 & 7994250 & 7986084 & 8166 & 250 \\
$\# 3$ & 7992166 & 7980326 & 11840 & 363 \\
$\# 4$ & 7993315 & 7973924 & 19391 & 594 \\
$\# 5$ & 7991294 & 7969530 & 21764 & 681 \\
\hline
\end{tabular}

\subsection{Experimental process}

First, pretreatment for the measurement experiment was carried out. Nitrogen is the main inert gas in the atmosphere, and it is reasonable to use it as a carrier gas. $\mathrm{N}_{2}$ was introduced into a small closed container at a rate of $150 \mathrm{ml} / \mathrm{min}$, then the output frequency of the QCM was allowed to stabilize at $0.01 \mathrm{~Hz} / \mathrm{s}$. As shown in Fig. 5, the sensor reached an equilibrium state.

After this equilibrium state lasted for about half an hour, we delivered the target gas into the container. Then, we waited for the sensor to reach a new balance before making subsequent measurements.

The main aim of the experiment was to evaluate the sensing performance of the gas sensor for acetone gas at RT. Two VOCs, butanol and isopropanol, were selected for comparison when evaluating the selectivity.

\section{Results and Discussion}

\subsection{Stability of QCM gas sensor}

Figure 6(a) shows the frequency deviation curve of each sensor over $30 \mathrm{~min}$ at RT. The concentration of acetone gas introduced was $1000 \mathrm{ppm}$, and the frequency fluctuation range of all sensors during this period was relatively small, indicating that the gas sensors have good stability.

We set the acetone gas concentration to $100 \mathrm{ppm}$, and the output frequency response of each sensor is shown in Fig. 6(b). The general trend can be seen from the figure: the frequency offset increases with the thickness of the sensitive film, indicating that the gas-sensing property of the GQD nanomaterial is related to the thickness of the coated film. However, when the 


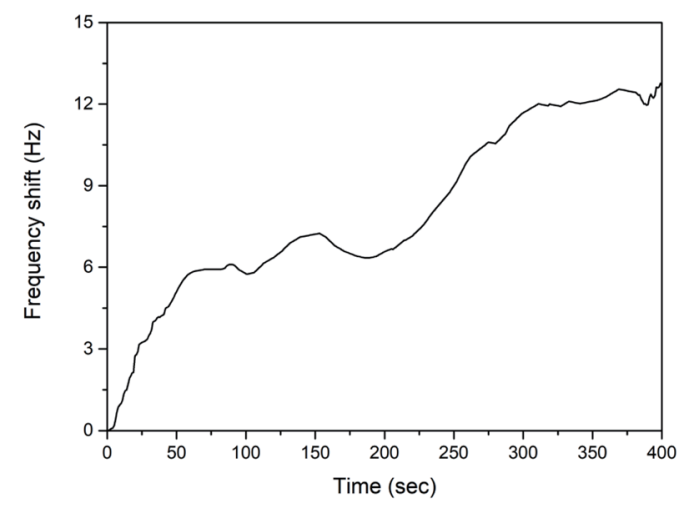

Fig. 5. Output frequency response when nitrogen was introduced.

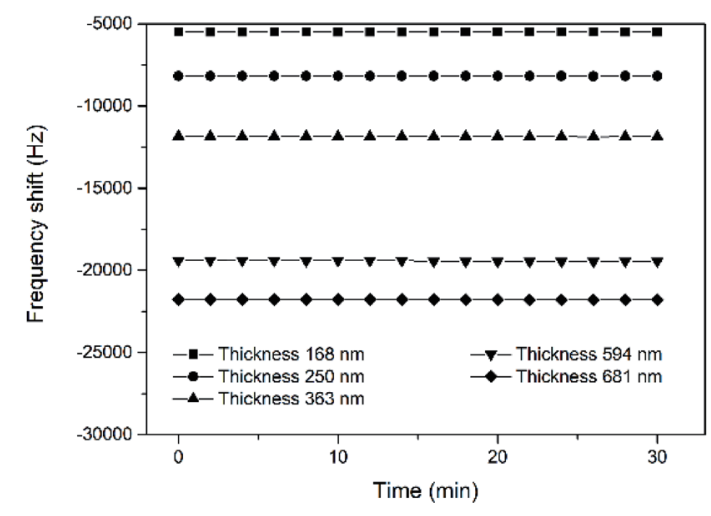

(a)

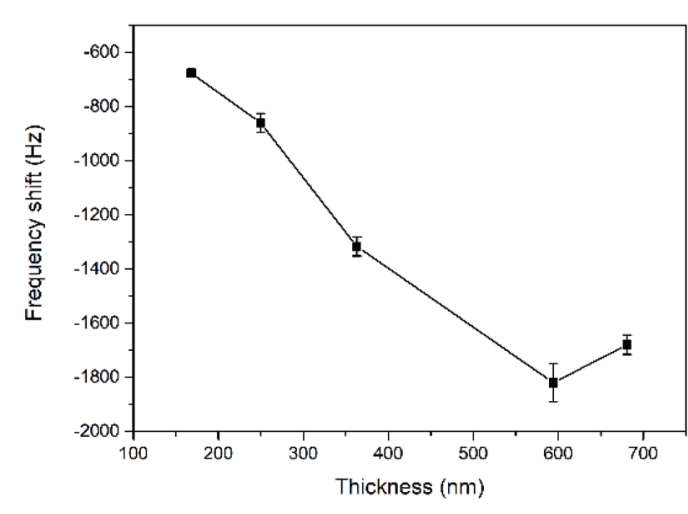

(b)

Fig. 6. Response frequency over time for sensors with films of different thicknesses. (a) Response of sensor sample frequency over time for sensors with films of different thicknesses. (b) Frequency output response curve of sensors with different film thicknesses at $100 \mathrm{ppm}$ acetone concentration.

thickness reaches a certain level, the response value saturates, indicating that the adsorption capacity of the film is saturated. On this basis, sensor \#4 (594 nm thickness) was selected for the measurement in all subsequent experiments.

\subsection{Sensitivity of QCM gas sensor}

In general, the output response, i.e., frequency shift, of the sensor increases with the acetone gas concentration (Figs. 7 and 8). When the acetone concentration is 120 and $240 \mathrm{ppm}$, the frequency shift of the output is 2280 and $4628 \mathrm{~Hz}$, respectively, indicating that the acetone sensor has good sensitivity at RT.

The fitting line of the frequency shift-acetone gas concentration curve [Fig. 8(b)] shows that the sensor has good linearity at concentrations of $2.5-240 \mathrm{ppm}$ acetone with a sensitivity of $16.78 \mathrm{~Hz} / \mathrm{ppm}$. The coefficient of determination $R^{2}$ of the fitting line is 0.95658 . Moreover, the minimum detection limit of the gas sensor for acetone is $2.5 \mathrm{ppm}$. 


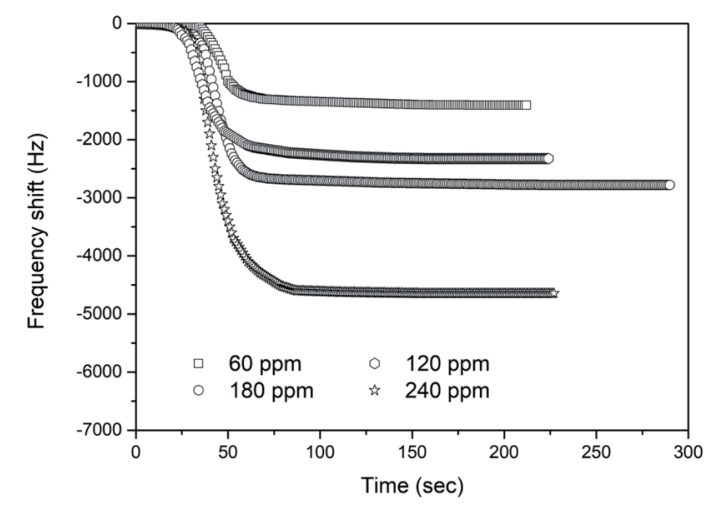

Fig. 7. Output response curves for different acetone concentrations.

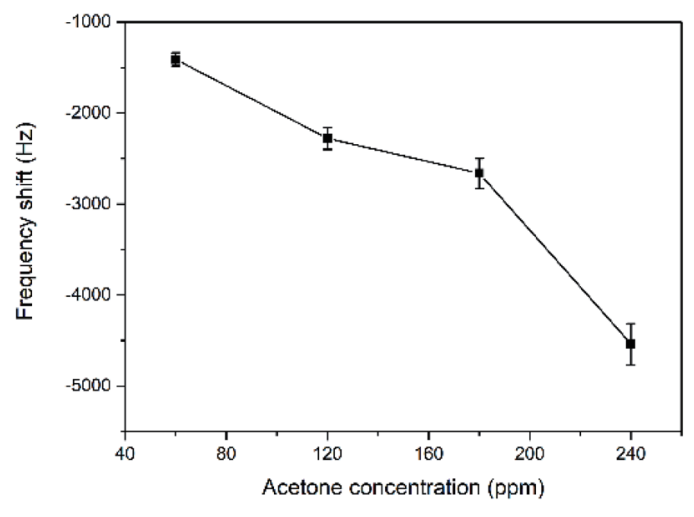

(a)

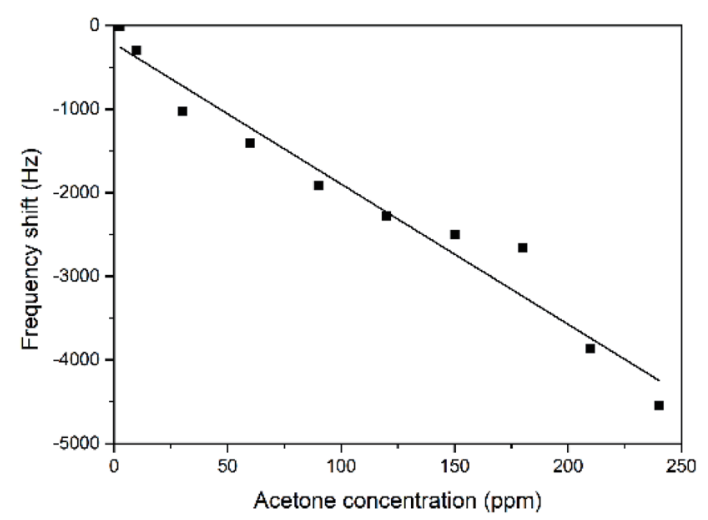

(b)

Fig. 8. Relationship between sensor response and acetone concentration. (a) Error bars of output response as a function of acetone concentration. (b) Fitting line of sensor response as a function of acetone concentration.

\subsection{Repeatability and response and recovery times of QCM gas sensor}

The concentration of acetone gas in the feed we set in this experiment was $240 \mathrm{ppm}$. During this process, the passage of acetone was intermittent. The output frequency response of multiple adsorption cycles was observed during the measurement. For the convenience of observation, five cycles were taken for analysis. It can be seen from the graph [Fig. 9(a)] that the five adsorption and desorption cycles were basically the same. When acetone was introduced into the experimental device, the sensor immediately responded and its output frequency started to decrease. When acetone was no longer introduced, the output response of the sensor started to increase toward zero. This phenomenon shows that the acetone sensor has good reproducibility and reversibility. Note that in each cycle, when the acetone was no longer introduced, the sensor did not undergo immediate desorption, but a short period of peak turbulence occurred. Here, the sudden increase in the weight of the wafer is understood to have an impact on the oscillating circuit, and then the acetone gas diffuses from the surface of the sensitive material into the interior, resulting in a steady state for a short period of time. 


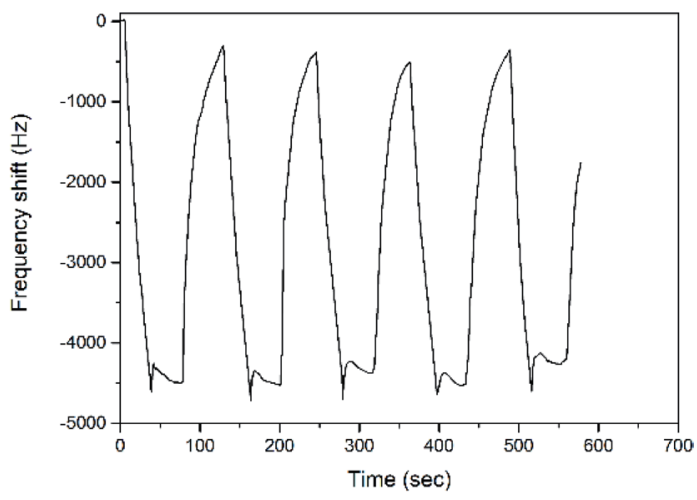

(a)

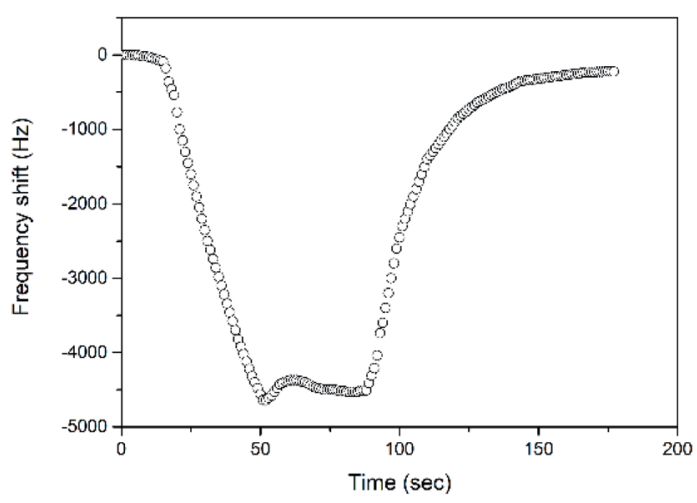

(c)

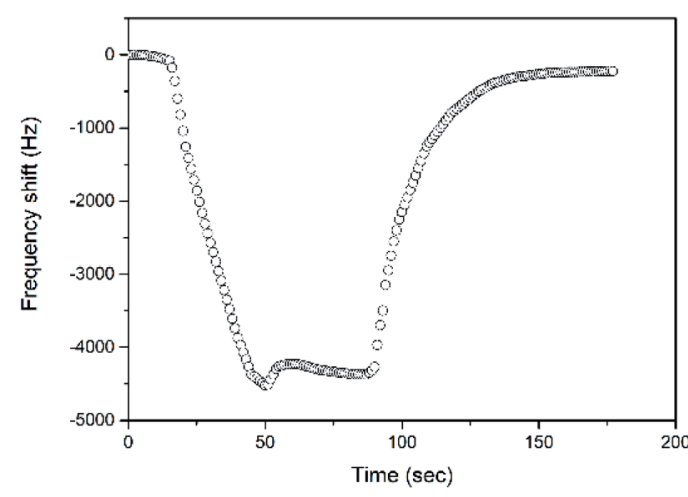

(b)

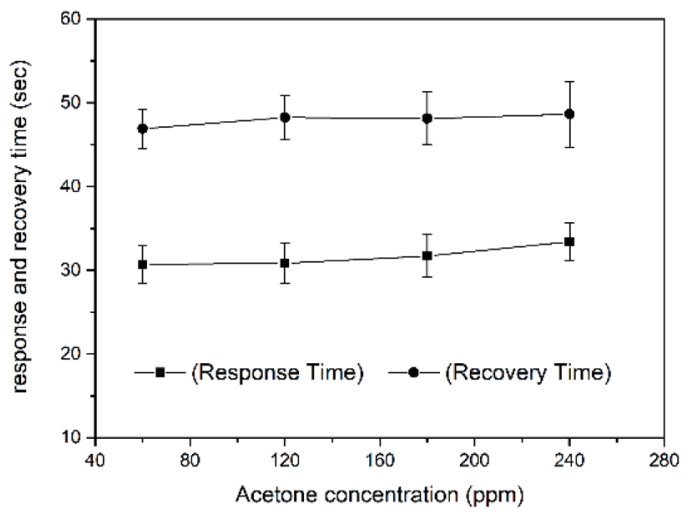

(d)

Fig. 9. Response and recovery times. (a) Gas sensor output response over time. (b) Response and recovery times in adsorption-desorption cycle. (c) Response and recovery times in adsorption-desorption cycle. (d) Response and recovery times plotted against acetone concentration.

In addition, we separately analyzed the two adsorption-desorption cycles in Figs. 9(b) and 9(c). We found that there is a slight difference between the times required for the adsorption and desorption processes, with the adsorption process generally slightly faster than the desorption process. Figure 9(d) shows the relationship between the acetone gas concentration and the response and recovery times. The response times required for adsorption and desorption in each cycle at different acetone gas concentrations are around 32 and $48 \mathrm{~s}$, respectively. That is, for the same sensor, the response speed is similar for different acetone concentrations. These results indicate that the acetone sensor has good sensitivity and repeatability.

\subsection{Selectivity of QCM gas sensor}

In the selectivity experiment, butanol and isopropanol were selected as comparison objects, the concentration of butanol and isopropanol was set to $1000 \mathrm{ppm}$, and the concentration of acetone gas was set to $100 \mathrm{ppm}$. It can be clearly seen (Fig. 10) that the sensor has a higher frequency output response to $100 \mathrm{ppm}$ acetone gas, which shows that the acetone sensor has good selectivity to the target gas. 


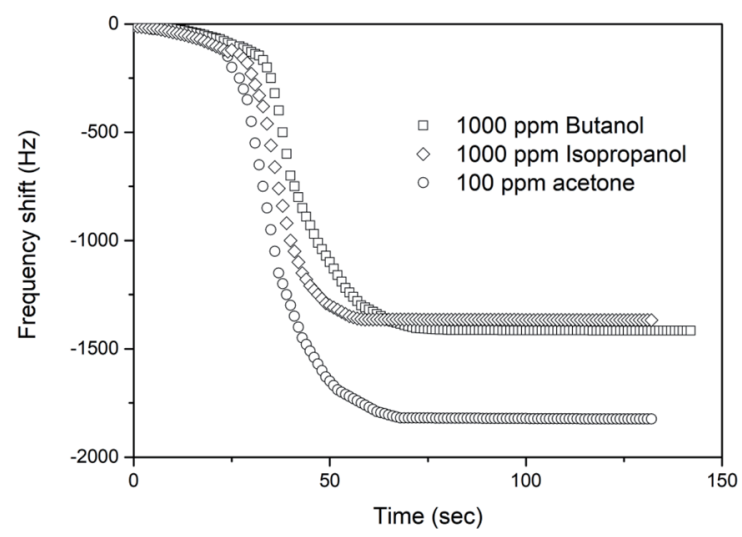

Fig. 10. Comparison of output responses for three VOCs.

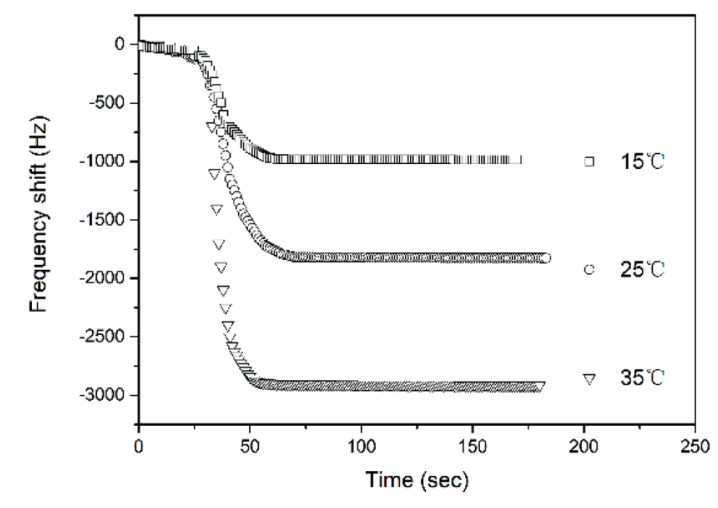

(a)

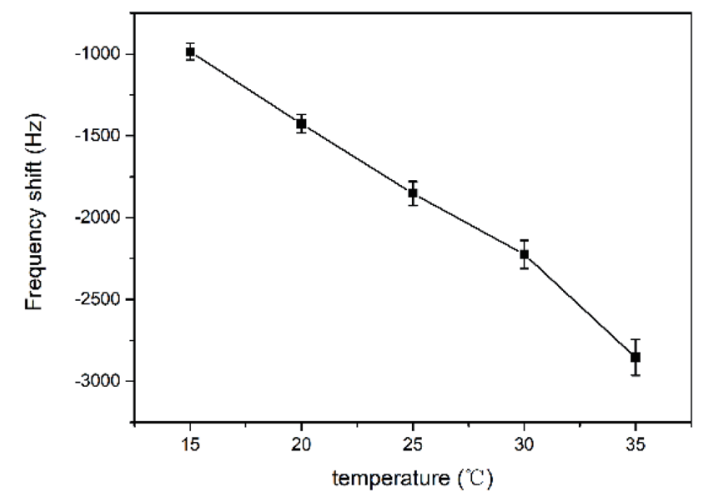

(b)

Fig. 11. Relationship between sensor response and temperature. (a) Output response plotted against time at different temperatures. (b) Error bars of the output response frequency at different temperatures.

\subsection{Impact of temperature}

Within the operating temperature range of 15 to $35^{\circ} \mathrm{C}$, for the same concentration of acetone gas $(100 \mathrm{ppm})$, the output frequency shift of the sensor increases with temperature [Figs. 11(a) and 11(b)], which indicates that the sensor has good sensitivity at temperatures suitable for the human body. In many studies, acetone gas sensors need to operate at high temperatures. Therefore, it is of practical significance to study acetone sensors with a good response at RT.

\subsection{Analysis of gas-sensing mechanism}

The gas-sensing mechanism in sensors is usually explained by the adsorption theory. Depending on the nature of the action of the surface molecules of the sensitive membrane on the target gas molecules, adsorption can be divided into physical adsorption and chemical adsorption. Physical adsorption generally refers to the van der Waals force, which is ubiquitous 
between all particles. Although the adsorption force itself is relatively weak, the specific surface area of the material and the structure of the adsorbent affect the degree of physical adsorption. In terms of gas-sensing properties, the interaction between the surface of the sensitive membrane and the target gas molecules is an important factor affecting the sensitivity and response speed. In other words, how the gas-sensitive film adsorbs the target gas affects the gas-sensing performance of the sensor. The surface of GQDs is rich in hydroxyl, carboxyl, and carbonyl groups and has a relatively large specific surface area as it is a nanomaterial. Its adsorption capacity is acceptable, so the material exhibits good sensitivity. The sensitive membrane adsorbs the gas to be tested in a percolation-diffusion process. At the moment of contact between the two, the acetone gas molecules cannot immediately and completely come in contact with the inner surface of all the pores and cracks of the sensitive film, and a buffering process is required. Therefore, in the repeatability experiment, we found that the sensor did not immediately desorb the gas molecules.

\section{Conclusions}

Acetone is a slightly toxic VOC. It is of practical significance to study acetone sensors operating at RT. In this paper, the gas-sensing properties of GQD nanomaterials based on a QCM sensing structure for acetone were investigated, including reproducibility, sensitivity, response time, selectivity, and stability. At a given temperature, the output frequency response of the sensor increases with the acetone concentration, but the response speed of the sensor is not affected by the target gas concentration. Several experiments examining the adsorptiondesorption cycles verified the good repeatability of the gas sensor. The sensor was found to exhibit excellent selectivity for acetone in the presence of two other VOCs. The present results demonstrated that GQDs are a promising sensing material for gas sensors for the detection of acetone at low concentrations.

\section{References}

1 K. He, J. Zhao, X. F Chu, W. J. Bi, W. Wang, C. Wang, and S. T. Liu: SC Advances 49 (2019) 28439.

2 V. Saasa, T. Malwela, M. Beukes, M. Mokgotho, C. P. Liu and B. Mwakikunga: Diagnostics 8 (2018)12.

3 W. T. Koo, J. S. Jang, S. J. Choi, and I. D. Kim: Acs Appl. Mater. Interfaces 21 (2017)18069.

4 A. Sett, M. Mondal, and T. K. Bhattacharyya: IEEE Sens. J. 19 (2019)3601.

5 T. V. Dang, H. N. Duc, D. N. Van, and H. N. Nguyen, Acs Appl. Mater. Interfaces 7 (2016)4828.

6 W. H. Jiang, L. L. Meng, S. F. Zhang, X. H. Chuai, P. Sun, F. M. Liu, X. Yan, Y. Gao, X. H. Liang, and G. Y. Lu: Microchimica. Acta 6 (2019) 186.

7 T. Hussain, H. Vovusha, R. Umer, and R. Ahuja, Appl. Surf. Sci. 456 (2018) 711.

8 Q. Jia, H. Ji, and X. Bai: J. Mater. Sci. Mater. Electron. 30 (2019) 7824.

9 X. Xu, Y. Chen, G. Zhang, S. Ma, Y. Lu, H. Bian, and Q. Chen, J. Alloys Compd. 703 (2017) 572.

10 Z. Jiang, M. Yin, and C .Wang: Mater. Lett. 194 (2017) 209.

11 M. S. Preethi, S. P. Bharath, and K. V. Bangera: Int. Conf. Advances in Mechanical Design (2018).

12 H. U. Ming-Jiang and J. Bing-Ying: Chinese J. Anal. Chem. 47 (2019) 363.

13 K. He, Z. Jin, X. Chu, and W. Bi: RSC Advances, 49 (2019) 28439.

14 J. Liang, R. Yang, K. Zhu, and M. Hu: J. Mater. Sci. Mater. Electron. 29 (2017) 3780.

15 W. Tao, P. Lin, Y. Ai, H. Wang, S. Ke, and X. Zeng: Anal. Biochem. 494 (2016) 85.

16 Q. Li, S. Zhang, L. Dai, L. Dai, and L. LI: J. Amer. Chem. Soc. 134 (2012) 18932.

17 P. K. Dewangan, F. Khan, K. Shrivas, and V. Sahu: J. Radioanal. Nucl. Chem. 320 (2019) 757. 
18 J. Peng, W. Gao, B. K. Gupta, Z. Liu, R. Romero-Aburto, L. Ge, L. Song, L. B. Alemany, X.Zhan, G. Gao, S. A. Vithayathil, B. A. Kaipparettu, A. A. Marti, T. Hayashi, J.Zhu, and P. M. Ajayan: Nano Lett. 12 (2012) 844.

19 S. Gwo, M.H. Lin, C. L. He, H. Y. Chen, and T. Teranishi: Langmuir 28 (2012) 8902.

20 Y. P. Sun, B. Zhou, Y. Lin, W. Wang, K. A. Fernando, P. Pathak, M. J. Meziani, B. A. Harruff, X. Wang, H. Wang, P. G. Luo, H. Yang, M. E. Kose, B. Chen, L. M. Veca, and S. Y. Xie: J. Am. Chem. Soc. 128 (2006) 7756.

21 A. Kaganskiy, S. Fischbach, A. Strittmatter, R. Sven, H. Tobias, and R. Stephan, Opt. Commun. 413 (2018) 162.

22 S. Haifeng, N. Yanli, F. Ruijie, and R. Ma: Ecotoxicol. Environ. Saf. 162 (2018) 10.

23 X. Wang, R. Li, Z. Li, and J. Liu: J. Colloid Interface Sci. 505 (2017) 847.

24 M. T. Hasan, R. Gonzalez-Rodriguez, C. Ryan, K, Pota, K. Green, J. L. Coffer, and A. V. Naumov: Nano Res. 5 (2019) 1041. 